\title{
1 Reconstructing the reproductive mode of an Ediacaran macro-organism
}

2 Mitchell, Emily G. ${ }^{1}$, Kenchington, Charlotte G. ${ }^{1,}$ Liu, Alexander G. ${ }^{2}$, Matthews, Jack J., ${ }^{3}$ and

3 Butterfield, Nicholas J. ${ }^{1}$

$4{ }^{1}$ Department of Earth Sciences, University of Cambridge, Downing Street, Cambridge, CB2

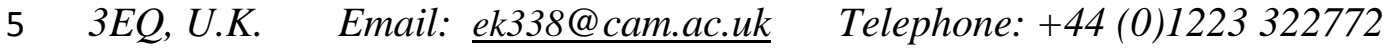

$6{ }^{2}$ School of Earth Sciences, University of Bristol, Life Sciences Building, 24 Tyndall Avenue,

$7 \quad$ Bristol, BS8 1TQ, U.K.

$8{ }^{3}$ Department of Earth Sciences, University of Oxford, South Parks Road, Oxford, OX1 3AN, $9 \quad U . K$.

11 Enigmatic macrofossils of late Ediacaran age (580-541 million years ago [Ma]) provide the oldest known record of diverse complex organisms on Earth, lying between the microbiallydominated ecosystems of the Proterozoic and the Cambrian emergence of the modern biosphere. ${ }^{1}$ Among the oldest and most enigmatic of these macrofossils are the Rangeomorpha, a group characterized by modular, self-similar branching and a sessile benthic habit. ${ }^{2,3,4}$ Localized occurrences of large in situ fossilized rangeomorph populations

17 allow fundamental aspects of their biology to be resolved using spatial point techniques. ${ }^{5}$ Here, we use such techniques to identify recurrent clustering patterns in the rangeomorph Fractofusus, revealing a complex life history of multigenerational, stolon-like asexual reproduction, interspersed with dispersal of waterborne propagules. Ecologically, such a habit would have allowed for both the rapid colonisation of a localized area and transport to new, previously uncolonized areas. The capacity of Fractofusus to derive adult morphology via two distinct reproductive modes documents the sophistication of its underlying developmental biology. 
Late Ediacaran sedimentary strata $(\sim 580-541 \mathrm{Ma})$ of Newfoundland and the UK are dominated by rangeomorphs, whose unique self-similar branching construction ${ }^{3}$ makes resolution of their phylogenetic relationships, or even their basic biology, difficult. ${ }^{1}$ The occurrence of rangeomorphs in conspicuously deep-water sediments has led to a general consensus that they were heterotrophic, ${ }^{6}$ while the global distribution of charniids (a rangeomorph sub-group) has been interpreted as evidence for reproduction via waterborne propagules. ${ }^{7}$ In the present study we use spatial statistics and modelling ${ }^{5,9}$ in a novel approach to illuminate the reproductive biology and underlying ecology of one of the most abundantly-preserved rangeomorph fossils, Fractofusus. ${ }^{8}$

We analysed three large bedding-plane assemblages of Fractofusus in SE Newfoundland: 1) the 'D' surface and 2) the 'E' surface at Mistaken Point, Avalon Peninsula; ${ }^{8,10}$ and 3) the H14 surface on Bonavista Peninsula (locality 14 of Hofmann et al.) ${ }^{11}$ (Extended data Fig. 1a-c). A volcanic tuff directly above the ' $\mathrm{E}$ ' surface has been dated to $565 \pm 3 \mathrm{Ma}$, ${ }^{12}$ which also constrains the age of the underlying ' $\mathrm{D}$ ' surface. Regional lithostratigraphic correlations suggest that the H14 surface is a few million years younger than the Mistaken Point beds. ${ }^{11}$ All three assemblages occur within deep-marine turbidite sequences, with Fractofusus fossils preserved as negative epirelief external moulds in siltstone hemipelagites, cast from above by volcaniclastic deposits. ${ }^{6}$

Fractofusus is conspicuously endemic, restricted almost exclusively to southeastern Newfoundland, ${ }^{13}$ where it dominates many macrofossil assemblages. ${ }^{10}$ Fractofusus has a rounded, elongate spindle-like morphology, with two (arguably three ${ }^{2,13}$ ) offset rows of irregularly alternating, self-similar, subdivided frondlets arranged along a central axis. ${ }^{2,14}$ Fractofusus specimens range from $1 \mathrm{~cm}$ to $42 \mathrm{~cm}$ in length ${ }^{2}$ (Fig. 1a,b); two species have been 
described, distinguished by their length:width ratios. ${ }^{2}$ The ' $D$ ' and ' $E$ ' surfaces are dominated by the elongate form, Fractofusus misrai (L/W=3.2, Fig. 1a), whereas the more ovate Fractofusus andersoni (L/W=1.6, Fig. 1b) dominates the H14 surface. ${ }^{10}$ Fractofusus occurs in dense benthic populations and exhibits no evidence of motility or current orientation. ${ }^{2}$ Together with nearest neighbour spatial analyses, ${ }^{10}$ these observations point to a sessile, recumbent, benthic mode of life in aggregated communities.

The spatial positions of Fractofusus were mapped to millimetre-scale resolution using differentiated GPS (Extended data Figs. 1d-f) on the two surfaces at Mistaken Point, and by tracing specimen outlines onto acetate sheets at H14; significantly, this latter approach also allowed size data to be recorded (Extended data Fig. 1f). The ' $D$ ' and 'E' surface data were corrected for tectonic deformation prior to analysis (Extended data Fig. 2). ${ }^{7}$ Heterogeneous Poisson models were used to identify possible distortions arising from differential erosion of the bedding planes (Supplementary Table 1). Pair correlation functions (PCF) were calculated to describe the spatial distributions of taxa on each bedding plane. ${ }^{5}$ Monte Carlo simulations ${ }^{15}$ and Diggle's goodness-of-fit test ${ }^{5}$ (the $\mathrm{p}$-value $p_{d}$, where $p_{d}=1$ indicates a perfect model fit and $p_{d}=0$ indicates no fit), were used to compare the fit of different spatial models to the data (specifically homogeneous and heterogeneous Poisson models ${ }^{16}$ and single and double homogeneous and heterogeneous Thomas cluster models). ${ }^{16}$ PCFs were also used to describe the spatial distributions of taxa other than Fractofusus on the 'D' and 'E' surfaces. For the H14 surface, spatial relationships between three distinct Fractofusus size classes (defined in Methods, Extended data Figs. 3a,b) were analysed by calculating partial $\mathrm{PCF}^{5}$ and comparing model fit of bivariate shared parents models (SP) with linked cluster models (LCM). ${ }^{16}$ Finally, spatial directionality was investigated by plotting their generalised K- 
functions ${ }^{17}$ from $0^{\circ}$ to $360^{\circ}$ (isotropy plots), allowing visualisation of the relative directional positions of specimens (Fig. 3).

Non-random spatial distributions of sessile organisms, i.e. those that do not exhibit complete spatial randomness (CSR), can be explained by either extrinsic factors (e.g. environmental heterogeneities), or intrinsic reproduction. ${ }^{18}$ Identifying the processes behind such patterns is not straight-forward; however, extrinsically-induced patterns are generally best modelled by heterogeneous Poisson models, ${ }^{18}$ which describe randomly distributed points with a nonuniform density across the sampled area. In contrast, intrinsic processes typically generate Thomas cluster models, ${ }^{18}$ where the points within each cluster have a normal density distribution centred on a parent point.

All three populations of Fractofusus were found to be significantly aggregated, conforming closely to homogeneous Thomas cluster models (Fig. 2a). Specimens on the 'E' and H14 surfaces are aggregated at two spatial scales, forming clusters of clusters (Fig. 2a,b). On the 'E' surface, this distribution is best modelled by a nested homogeneous double Thomas cluster model of 23 clusters (radius $r=0.242 \mathrm{~m}$ ), each containing 12 smaller clusters $(r=0.074 \mathrm{~m})$ of 3 specimens $\left(p_{d}=0.76\right)$. The H14 surface distribution is best modelled by a nested homogeneous double Thomas cluster model of 24 large clusters $(r=0.237 \mathrm{~m})$, each containing 6 clusters $(r=0.079 \mathrm{~m})$ of 8 specimens $\left(p_{d}=0.89\right)$. The ' $\mathrm{D}$ ' surface distribution forms discrete clusters (not clusters of clusters), which are best modelled by a single Thomas cluster model $\left(p_{d}=0.77\right)$ with 338 Fractofusus clusters of 3 specimens $(r=0.086 \mathrm{~m})$ (Extended data Tables 1-2). Importantly, the spatial distribution on the 'E' surface can also be modelled by the nested double cluster pattern found on the H14 surface (Fig. 2b) $\left(p_{d}{ }^{H}\right.$ on ${ }^{E}=0.51$ ), strongly implying the same underlying process for both distributions (Fig. 2b, 
99 Extended data Table 4). The spatial distribution of Fractofusus on the 'D' surface is conspicuously similar to that seen in the larger specimens on H14 (Extended data Fig. 4e). By contrast, the spatial distributions of other taxa - Thectardis, Primocandelabrum and Charniodiscus - exhibit fundamentally different magnitudes and spatial scales of aggregation, both to each other and to those of Fractofusus (Fig. 2b and Extended data Tables $3,5)$.

The close fit of Fractofusus spatial distributions to single and nested double Thomas cluster models strongly suggests that they derive from reproductive rather than extrinsic (environmental) factors. Reproductive biology is further corroborated by size analysis of the Fractofusus population on the H14 surface (Fig. 2c, Extended data Figs. 4a,c,d), which reveals strikingly different spatial patterns for each of the three size classes (Fig. 2c, Extended data Tables $1-2)$. Whereas the largest size class $(>11.0 \mathrm{~cm})$ is randomly distributed $\left(p_{d}=0.30\right)$, both the intermediate $(5.5-11.0 \mathrm{~cm})$ and smallest $(<5.5 \mathrm{~cm})$ size classes are hierarchically clustered: small individuals cluster around intermediate individuals $\left(p_{d}{ }^{L C M}=0.74\right.$ versus $p_{d}^{S P}=0.03$; Extended data Table 5), which in turn cluster around large individuals $\left(p_{d}{ }^{L C M}=0.66\right.$ versus $\left.p_{d}^{S P}=0.01\right)$. In other words, the smallest specimens form clusters (homogeneous nested double Thomas cluster model $\left(p_{d}=0.72\right)$ ) around intermediatesized specimens (homogeneous single Thomas cluster model; $p_{d}=0.51$ ), which are themselves clustered around randomly distributed large specimens (homogeneous Poisson model $p_{d}=0.31$; Figs. 2c, 4, Extended data Fig. 5, Extended data Tables 1-2). Moreover, the isotropy plots for H14 (Fig. 3) show strong directionality for the large size class, but limited directionality for the medium and small size classes. The nested clusters on the 'E' and H14 surfaces suggest three generations, while the single clusters on the ' $\mathrm{D}$ ' surface suggest two, reflecting an earlier stage in population development. The difference is consistent with the 
suggestion that the ' $\mathrm{D}$ ' surface records an earlier stage in the ecological succession of Ediacaran macroscopic communities ${ }^{10}$ (SI:2:3).

As with other Ediacaran macrofossils, there is no direct fossil evidence of reproductive habits in Fractofusus, but its recurrent distribution on bedding surfaces provides a statistically robust approach for inferring the underlying processes. ${ }^{19}$ In modern oceans, large sessile organisms typically reproduce by means of waterborne propagules, fragmentation/budding, and/or stolons (i.e., production of asexual clones that are at least initially connected to the parent by specialized outgrowths).

Spatial distribution of waterborne propagules - including both sexual and asexual spores, as well as sub-millimetre buds and fragments - are a function of current and rate of sinking. Even with rapid sinking $(\sim 1 \mathrm{~mm} / \mathrm{s})^{20}$ and slow currents $(\sim 1 \mathrm{~cm} / \mathrm{s})$, propagules released from the dorsal surface of a 'parental' Fractofusus ( $2-3 \mathrm{~cm}$ above the substrate) would have been current aligned ${ }^{21}$ and dispersed by decimetres or more. ${ }^{22}$ Slow descent times also correspond with right-skewed (mean greater than the median) density distributions. ${ }^{20,21}$ The random spatial distribution of the H14 largest size class likely reflects a large dispersal distance (Extended data Table 1), which coupled with its highly directional isotropy plot (Fig. 3a) indicates that the largest specimens were strongly influenced by currents (c.f. Darroch et al. $^{7,10}$ ) As such, they likely derive from waterborne propagules and represent the initial establishment of a Fractofusus population on this surface.

The hierarchically clustered bedding plane distributions of small and medium Fractofusus on H14 closely match patterns exhibited by organisms reproducing asexually via stolon-like lateral extensions (Extended data Fig. 5). ${ }^{23}$ Cluster distributions of the small and medium 
size classes are also highly left-skewed (median greater than the mean), with the mean distance from each "parent" to their "offspring" on the order of a few centimetres (Fig. 2a), and offspring exhibiting no significant directionality or current orientation (Fig. 3b-c,

152 Extended data Fig. 3c). The reproducibility of the model distributions across the three bedding-plane assemblages further attests to the indifferent effects of current: the spatial distributions of non-tethered offspring would result in patterns dependent on current velocity, which are unlikely to be consistent across multiple bedding planes in different localities. Moreover, there are no recorded instances of buds or fragmentary specimens of Fractofusus in any of its $5000+$ documented specimens ${ }^{1,10,11,24}$ (see SI:2.5, SI:3). As such, the Fractofusus clusters on the H14 surface are not consistent with waterborne propagules or fragmentation/budding, but are directly comparable to stolon-like reproduction. Other taxa exhibit an intriguing range of non-random habits, and our preliminary analyses indicate that Primocandelabrum and Charniodiscus may have also reproduced using stolons.

Reproductive biology lies at the core of ecological and evolutionary dynamics, and its positive identification in Ediacaran macrofossils has the potential to illuminate the beginnings of the modern marine biosphere. Previous studies of Ediacaran macrofossils have investigated the seasonality of reproduction ${ }^{7}$, identified putative stolons ${ }^{28,29}$, and inferred sexual or asexual reproduction based on biogeographic distribution or qualitative description of local populations. ${ }^{7,10}$ In the case of phosphatized 'embryo' microfossils, internal cell packages have been interpreted as evidence of germ-soma differentiation, ${ }^{30}$ but it remains to be seen how those fossils relate to the evolution of large and/or complex eukaryotes.

172 The identification in Fractofusus of a multigenerational asexual clonal phase, interspersed 173 with the release of waterborne propagules, is the first statistically robust account of 
reproductive life history reported in an Ediacaran macrofossil. Such a strategy would have allowed for the rapid exploitation of localized areas, as well as for transport to new, previously uncolonized areas. The conclusion that Fractofusus could switch between reproductive modes further reveals the sophistication of its underlying developmental programme, capable not only of tissue differentiation, but also the generation of new macroscopic individuals from both benthic stolons and waterborne propagules.

\section{References}

[1] Liu, A. G, Kenchington, C. G. \& Mitchell, E. G. Remarkable insights into the paleoecology of the Avalonian Ediacaran biota. Gondwana Research 27:4 1355-1380 (2015) [2] Gehling, J. G. \& Narbonne, G. M. Spindle-shaped Ediacara fossils from the Mistaken Point assemblage, Avalon Zone, Newfoundland. Canadian Journal of Earth Sciences 44(3), 367-387 (2007)

[3] Narbonne, G. M. Modular construction of early Ediacaran complex life forms. Science 305(5687), 1141-1144 (2004)

[4] Hoyal Cuthill, J. F. \& Conway Morris, S. Fractal branching organizations of Ediacaran rangeomorph fronds reveal a lost Proterozoic body plan. PNAS 111, 13122$13126(2014)$

[5] Illian, J., Penttinen, A., Stoyan, H. \& Stoyan, D. Statistical analysis and modelling of spatial point patterns (Vol. 70). John Wiley \& Sons. 560 p. (2008)

[6] Wood, D. A., Dalrymple, R. W., Narbonne, G. M., Gehling, J. G. \& Clapham, M. E. Paleoenvironmental analysis of the late Neoproterozoic Mistaken Point and Trepassey formations, Southeastern Newfoundland. Canadian Journal of Earth Sciences 40, 1375-1391 (2003) 

oldest known macroscopic communities from Mistaken Point, Newfoundland. Paleobiology 39, 591-608 (2013)

201

[8] Landing, E., Narbonne, G. M. \& Myrow, P. Trace fossils, small shelly fossils and the Precambrian-Cambrian boundary. University of the State of New York, 81 p. (1988)

[9] Wiegand, T., Gunatilleke, S., Gunatilleke, N. \& Okuda, T. Analyzing the spatial structure of a Sri Lankan tree species with multiple scales of clustering. Ecology 88, 3088$3102(2007)$

[10] Clapham, M. E., Narbonne, G. M. \& Gehling, J. G. Paleoecology of the oldest known animal communities: Ediacaran assemblages at Mistaken Point, Newfoundland.

Paleobiology 29, 527-544 (2003)

[11] Hofmann, H. J., O’Brien, S. J. \& King, A. F. Ediacaran biota on Bonavista

Peninsula, Newfoundland, Can. J. of Paleontology 82, 1-36 (2008)

[12] Benus, A. P. Sedimentological context of a deep-water Ediacaran fauna (Mistaken

Point Formation, Avalon zone, eastern Newfoundland). Bull. NY State Mus. 463, 8-9

[13] Narbonne, G. M., Laflamme, M., Trusler, P. W., Dalrymple, R. W. \& Greentree, C.

Deep-water Ediacaran fossils from northwestern Canada: Taphonomy, ecology, and evolution. J. of Paleontology 88(2), 207-223 (2014)

[14] Brasier, M. D., Antcliffe, J. B. \& Liu, A. G. The architecture of Ediacaran fronds. Palaeontology 55(5), 1105-1124 (2012)

[15] Diggle, P. Statistical analysis of spatial point patterns, 2nd ed. 148 pages. Arnold, London (2003)

[16] Baddeley, A. \& Turner, R. Practical maximum pseudolikelihood for spatial point patterns. Australian \& New Zealand Journal of Statistics 42, 283-322 (2000) 
[17] Chiu, S. N., Stoyan, D., Kendall, W. S., \& Mecke, J. Stochastic geometry and its applications. John Wiley \& Sons. pgs 570 (2013).

[18] Lin, Y., Chang, L., Yang, K., Wang, H. \& Sun, I. Point patterns of tree distribution determined by habitat heterogeneity and dispersal limitation. Oecologia 165, 175-184 (2011)

[19] Droser, M. L., \& Gehling, J. G. Synchronous aggregate growth in an abundant new Ediacaran tubular organism. Science 319(5870), 1660-1662 (2008)

[20] Gaylord, B., Reed, D. C., Raimondi, P. T. \& Washburn, L. Macroalgal spore dispersal in coastal environments: mechanistic insights revealed by theory and experiment. Ecological Monographs 76(4), 481-502 (2006)

[21] Shanks, A. L. Pelagic larval duration and dispersal distance revisited. The Biological Bulletin 216(3), 373-385 (2009)

[22] Gaylord, B., Reed, D., Raimondi, P., Washburn, L. \& McLean, S. A physically based model of macroalgal spore dispersal in the wave and current-dominated nearshore. Ecology 83, 1239-1251 (2002)

[23] Araki, K., Shimatani, K. \& Ohara, M. Dynamics of distribution and performance of ramets constructing genets: a demographic-genetic study in a clonal plant, Convallaria keiskei. Annals of Botany 1-9 (2009)

[24] Narbonne, G. M. \& Gehling, J. G. Life after snowball: the oldest complex Ediacaran fossils. Geology 31, 27-30 (2003)

[25] Penny, A. M. et al. Ediacaran metazoan reefs from the Nama Group, Namibia. Science 344(6191), 1504-1506 (2014)

[26] Yuan, X. et al. The Lantian biota: a new window onto the origin and early evolution of multicellular organisms. Chinese Science Bulletin 58(7), 701-707 (2013) 

reproduction in the earliest biomineralizing animal Cloudina. Geology 33(4), 277-280 (2005)

Peterson, K. J., Waggoner, B. \& Hagadorn, J. W. A fungal analog for

Newfoundland Ediacaran fossils? Integrative and Comparative Biology 43, 127-136 (2003)

[29] Fedonkin, M. A. Sistematicheskoye opisaniye vendskikh Metazoa, p. 70-106. In B.

S. Sokolov \& A. B. Ivanovskiy (eds.), Vendskaya sistema 1, istoriko-geologicheskoe i soma separation in Ediacaran animal embryo-like fossils, Nature 516, 238-241 (2014)

\section{Supplementary Information}

Extended Data

260

Supplementary Methods

261

Supplementary Information

262

Supplementary Data

263

264

\section{Acknowledgements}

The Parks and Natural Areas Division, Department of Environment and Conservation,

Government of Newfoundland and Labrador provided permits to conduct research within the Mistaken Point Ecological Reserve in 2010, while the Department of Tourism, Culture and Recreation provided permits for paleontological research on the Bonavista Peninsula in 2012. Readers are advised that access to both of the aforementioned fossil localities is by scientific research permit only. Contact the relevant Department listed above for further information.

271 This work has been supported by the Natural Environment Research Council [grant numbers 
272 NE/I005927/1 to C.G.K., NE/J5000045/1 to J.J.M., NE/L011409/1 to A.G.L. and

273 NE/G523539/1 to E.G.M.], and a Henslow Junior Research Fellowship from Cambridge

274 Philosophical Society to A.G.L. We thank Marc Laflamme for discussions on this

275 manuscript.

276

277 Author Contributions

278 E.G.M conceived the project, collected data on the ' $D$ ' and ' $E$ ' surfaces and ran the analyses.

279 C.G.K, A.G.L and J.J. M collected data on the H14 surface. All authors discussed the results 280 and prepared the manuscript.

281

282 Author Information

283 Department of Earth Sciences, University of Cambridge, Downing Street, Cambridge, CB2 284 3EQ, U.K.

285 Emily G. Mitchell, Charlotte G. Kenchington, and Nicholas J.Butterfield

286 School of Earth Sciences, University of Bristol, Life Sciences Building, 24 Tyndall Avenue, 287 Bristol, BS8 1TQ, U.K.

288 Alexander G. Liu

289 Department of Earth Sciences, University of Oxford, South Parks Road, Oxford, OX1 3AN, 290 U.K.

291 Jack J. Matthews

292 


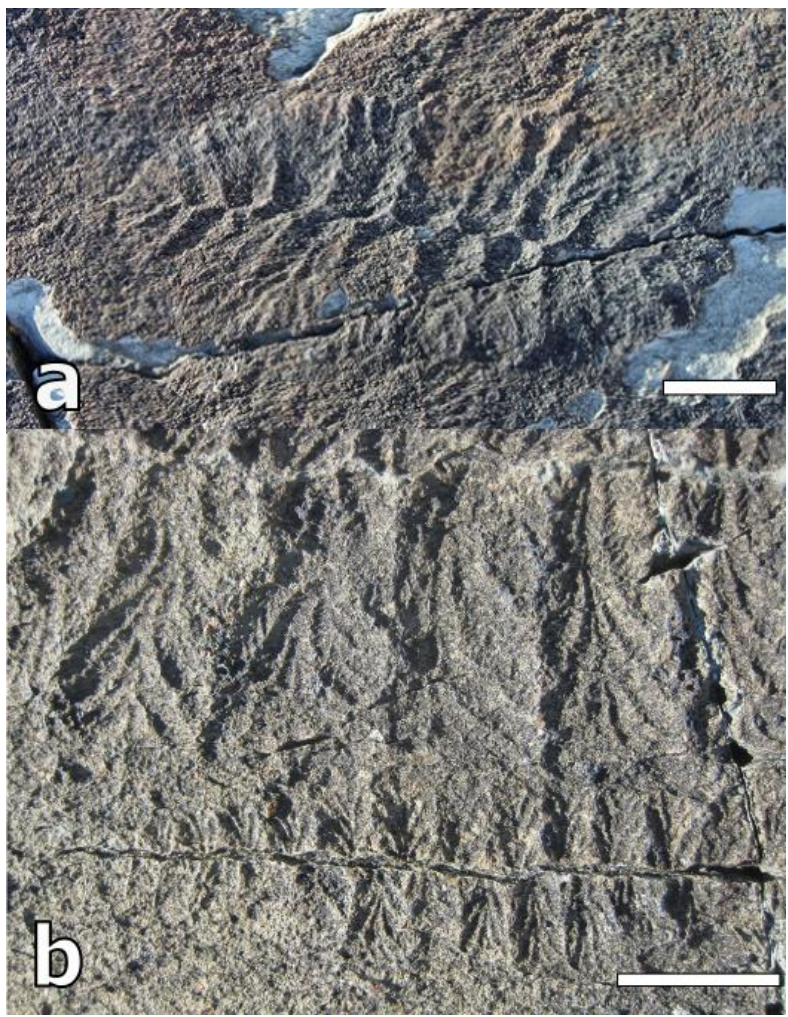

295 Figure 1: a, Fractofusus andersoni specimen from the H14 surface. b, Fractofusus misrai

296 from the 'E' surface, showing a large size-class partial specimen ( 20cm, above) alongside a

297 small size-class specimen $(3.5 \mathrm{~cm}$ in length, below). Scale bars $=1 \mathrm{~cm}$. Photographs are

298 unretrodeformed. 

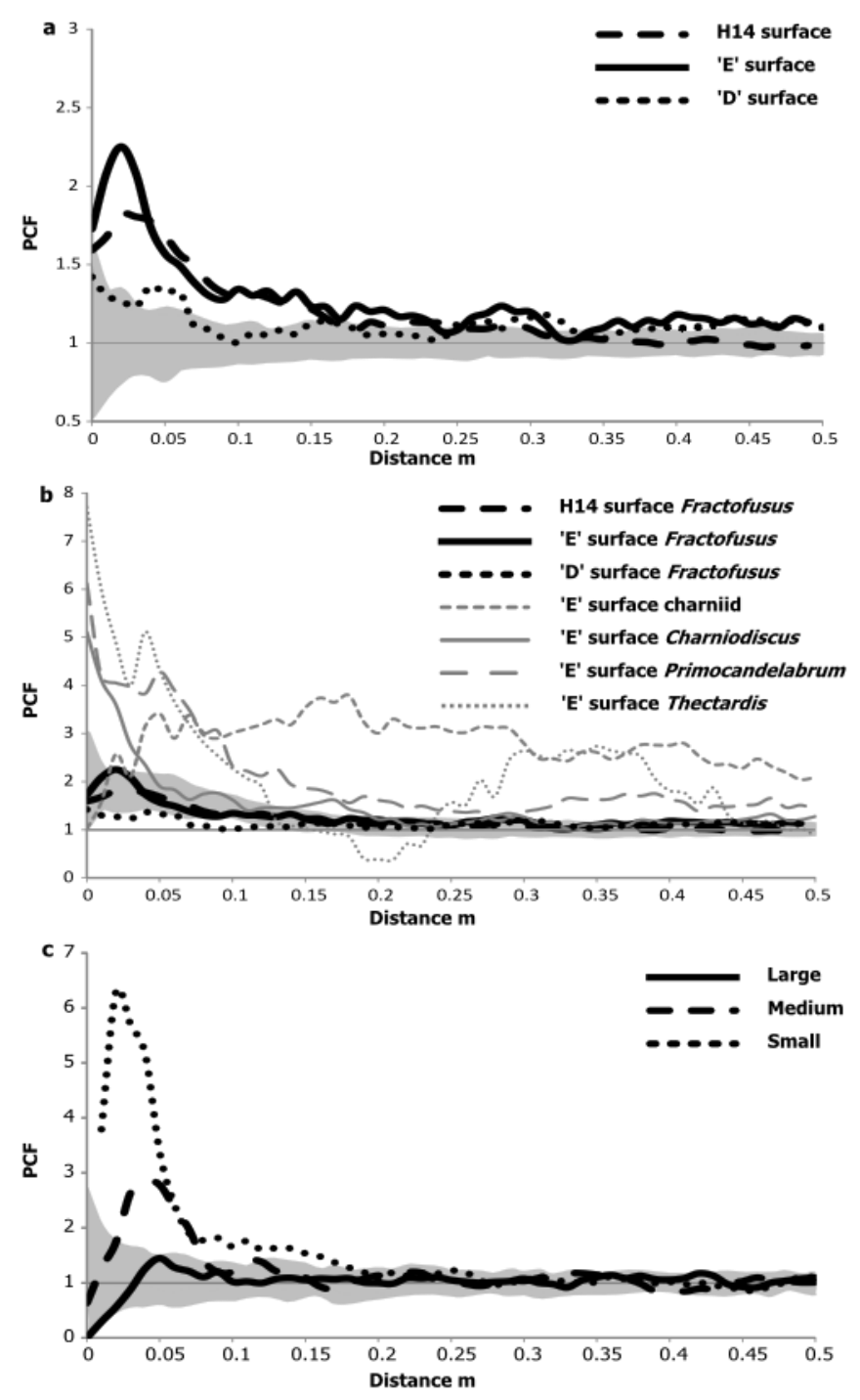

Figure 2: PCF for mapped taxa. For all plots the $\mathrm{x}$-axis is the inter-point distance between organisms in metres. The $\mathrm{y}$-axis $\mathrm{PCF}=1$ indicate $\mathrm{CSR},<1$ indicates segregation, and $>1$ indicates aggregation. a, PCF for Fractofusus on the 'D' surface (1040 specimens), 'E' surface (1141 specimens) and H14 surface (1214 specimens). Grey shaded area depicts the bounds of 99 Monte Carlo simulations of CSR. Since the PCF curves are not completely within these areas, the CSR hypothesis is rejected and one can assume that the Fractofusus distributions on all three surfaces form cluster patterns $\left(p^{D}{ }_{d}<0.01, p^{E}{ }_{d}<0.01, p^{H 14}{ }_{d}<0.01\right)$. b, PCF for non-CSR 'E' surface taxa (charniid 76 specimens, Charniodiscus 326 specimens, 99 Monte Carlo simulation of the best-fit H14 surface model of double Thomas cluster 

process. Note how the 'E' surface Fractofusus PCF follows the H14 surface PCF very 311 closely, and can be modelled by the same process $\left(p_{d}=0.51\right)$. Other ' $E$ ' surface taxa have 312 dramatically different PCF to the Fractofusus PCF. c, PCF for the three size classes of 313 Fractofusus on H14 surface. Grey shaded area depicts the 99 Monte Carlo simulation of CSR 314 . The large size-class (350 specimens) exhibits CSR $\left(p_{d}=0.30\right)$, the intermediate size-class 315 (310 specimens) shows aggregation $<0.10 \mathrm{~m}$ (single Thomas cluster model $\left(p_{d}=0.51\right)$ ). The 316 small size-class (554 specimens) shows a large aggregation $<0.08 \mathrm{~m}$ and a lesser aggregation 317 between $0.08 \mathrm{~m}$ and $0.20 \mathrm{~m}$ (double Thomas cluster model $\left(p_{d}=0.72\right)$ ). 

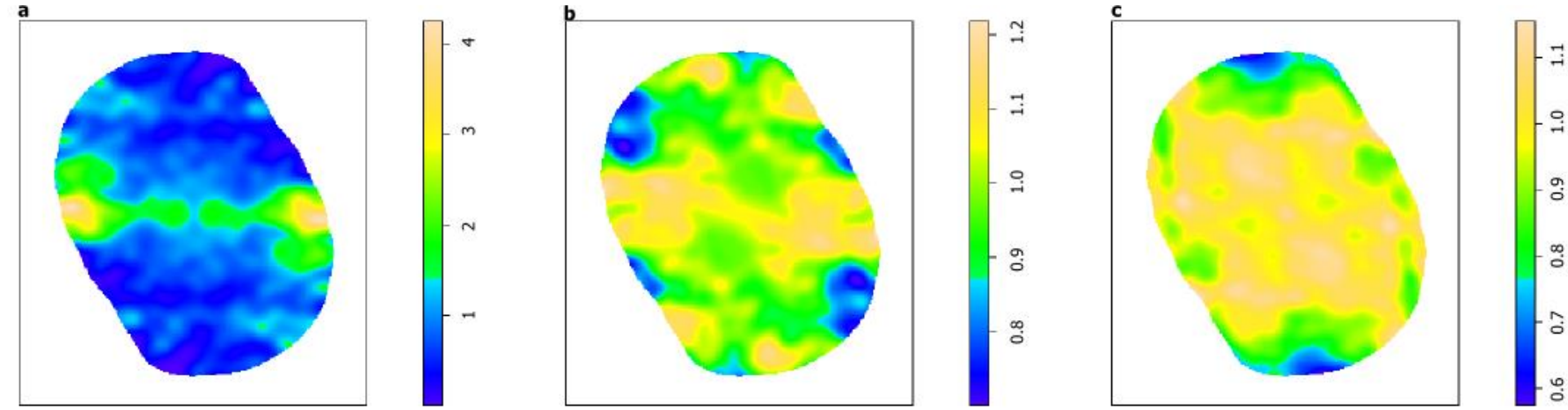

321

322

323

324

325

326

327

328

329

330

331

332

333

334

Figure 3. Isotropy plots from the H14 surface for each size class of Fractofusus, providing a visualisation of specimen positions relative to one another. The vertical axis on each subfigure depicts the colour map of specimens $/ \mathrm{m}^{2}$ normalised to account for different densities between size-classes. A peak $(<1)$ is shown in green or yellow and depicts clustering, while a dip $(<1)$ is shown in blue and depicts segregation. If there are no directional effects then the colour map in every direction from the centre point should be similar. a, The large size-class shows strong anisotropy, with aggregation of up to 4 normalised specimens $/ \mathrm{m}^{2}$. In contrast the $\mathbf{b}$, medium and $\mathbf{c}$, small size-classes show isotropy, that is a relative evenness of aggregations with a maximum density variation up to 0.5 normalised specimens $/ \mathrm{m}^{2}$. 

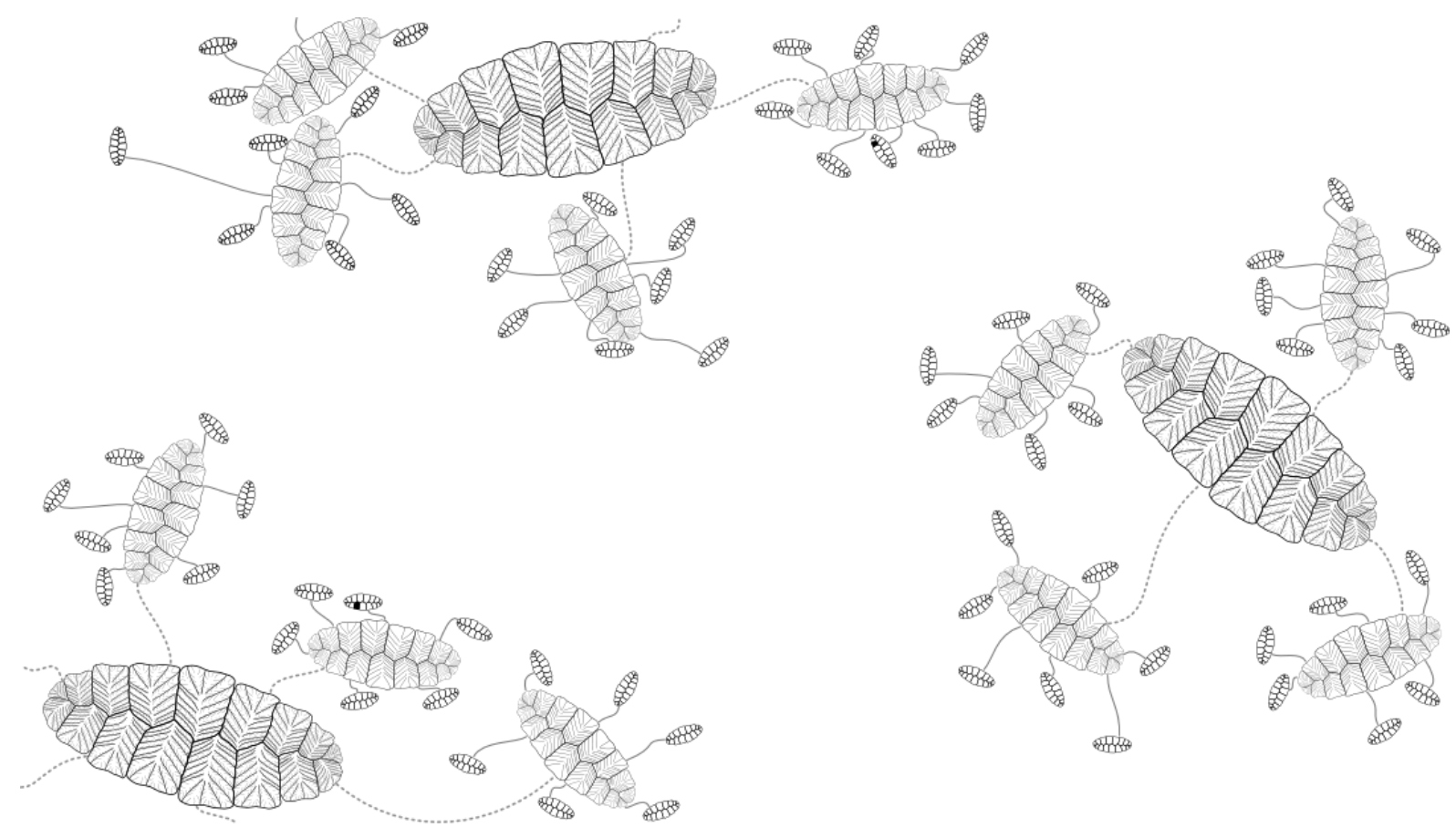

Figure 4: Schematic diagram showing simplified Fractofusus spatial arrangements. The actual number of clusters, and clusters within those clusters, is higher than shown ( 23 clusters each containing 12 clusters of 3 specimens on the H14 surface), making their direct visual detection challenging. No overlapping specimens are shown because, while the best-fit models allow for overlaps, the observed PCF between the small size-class (Extended data Fig. $4 \mathrm{c}, \mathrm{d})$ and the large size-class (Fig. $2 \mathrm{~b})$ shows a small segregation $(<3 \mathrm{~cm})$ away from the model behaviour, and a similar, non-significant segregation for the large size-class.

Extended Data Figure 1: Map and simplified stratigraphic column showing the position of studied bedding planes with bedding plane maps of Fractofusus. a, Newfoundland,

351 Eastern Canada. Dashed area indicates region of interest in B. b, The Avalon and Bonavista Peninsulas, eastern Newfoundland. Locations of the bedding planes are indicated. c, 
Stratigraphic column (not to scale) compiled of information from the Avalon and Bonavista

Peninsulas; lithological units in each region are treated as correlative in this study, but work

is ongoing to determine the validity of this assumption. The ' $E$ ' surface at Mistaken Point has been dated to $565 \pm 3 \mathrm{Ma}^{12}$. There are currently no available radiometric dates from the Bonavista Peninsula. Maps of Fractofusus positions on d, the 'D' surface, e, the 'E' surface and $\mathbf{f}$, the H14 surface. In Fig. e the largest specimens in light blue, medium specimens in mid blue and smallest specimens in dark blue.

Extended Data Figure 2: Retrodeformation calculations on the Mistaken Point surfaces.

Plots of the lengths versus widths of discs from a, the ' $D$ ' surface, Mistaken Point and $\mathbf{b}$, the 'E' surface Mistaken Point. The gradient of the line defines the retrodeformation factor, which for ' $D$ ' surface is $1.35 \pm 0.11\left(R^{2}=0.92\right)$, and for ' $E$ ' surface is $1.71 \pm 0.08\left(R^{2}=0.75\right)$. c, Fractofusus PCF on the 'E' surface with (solid black line) and without (dashed black line) retrodeformation. The grey shaded area depicts the boundary of 99 Monte Carlo simulations for the model which provided the best-fit model to the retrodeformed data, which has a good fit on the non-retrodeformed data $\left(p_{d}=0.60\right)$

Extended Data Figure 3: Size distribution analysis of Fractofusus for the H14 surface.

a, Size-frequency distributions for Fractofusus, and b, the results of Bayesian Information Criterion $^{52,53}$ (BIC) (univariate data). Triangles and squares correspond to models assuming equal and unequal variance respectively. High BIC values correspond to a good model fit, so the best-fit model is a three component equal variance model using log-normalized length data. c-d, Rose diagrams plotting the directional orientation of the different size classes of Fractofusus on H14 surface showing c, Large size class $(<11.0 \mathrm{~cm}), \mathbf{d}$, Intermediate size class $(5.5-11.0 \mathrm{~cm})$ and $\mathbf{e}$, Small size class $(<5.5 \mathrm{~cm})$. The angles of the Fractofusus central axis relative to North $\left(0^{\circ}\right)$. There is no strong orientation preference for any of the size classes. 

plots the $\mathrm{x}$-axis is the inter-point distance between organisms in metres. a, Mark correlation function, ${ }^{5}$ where 1 corresponds to a lack of correlation of size, such that Fractofusus size is independent and identically distributed. $<1$ corresponds to a positive dependency (in contrast to PCF) and $>1$ corresponds to a negative dependency. Fig. a shows that small Fractofusus on the H14 surface $(<0.3 \mathrm{~cm})$ are more likely to be found near each other than expected by random. b, The H14 surface PCF (solid line) showing the model that fits the data best, a double Thomas cluster model (dotted line, $p_{d}=0.89$ ), and the simulation envelope for 99 Monte Carlo simulations (grey shaded area). PCF for the best-fit models for the bivariate size-classes of Fractofusus on H14 surface showing: c, Linked cluster model for small with medium size classes $\left(p_{d}=0.74\right)$ and $\mathbf{d}$, Linked cluster model for medium with large size class $\left(p_{d}=0.66\right)$. e, The PCF of the largest size class of H14 (solid line), showing the CSR Monte

Carlo simulation envelope in grey, with the ' $\mathrm{D}$ ' surface PCF (dotted line, $p_{d}=0.56$ ). $\mathbf{f}$,

Nearest neighbour distances (solid line, $p_{d}=0.01$ ) with CSR Monte Carlo simulation envelope in grey.

Extended Data Figure 5: Artistic reconstruction of Fractofusus on the H14 surface, around which there are five to eight medium specimens clustered. Each of the medium specimens also has small specimens clustered around them. The small specimens therefore form an independent double cluster pattern, that is, clusters of clusters.

Extended Data Table 1: Best-fit univariate cluster models. For the heterogeneous backgrounds, the moving window radius is $0.5 \mathrm{~m}$ using the same taxon density as the taxon being modelled. $p_{d}=1$ corresponds to a perfect fit of the model on the data, while $p_{d}=0$ 
corresponds to no fit. The error function of the best-fit model gives the fraction of the total sum of squares for the transformed empirical PCF which are not explained by the model.

Extended Data Table 2: Best-fit univariate double cluster models. Large-scale clusters are determined for the univariate cluster then input into the model, and the small-scale clusters are determined in the double cluster analysis. $p_{d}=1$ corresponds to a perfect fit of the model on the data, while $p_{d}=0$ corresponds to no fit.

\section{Extended Data Table 3: Best-fit univariate cluster models on heterogeneous}

backgrounds for ' $\mathbf{E}$ ' surface taxa. Univariate clusters, either fitted to the small scale (S) or large scale (L) were modelled on different backgrounds defined by the density map of all taxonomic groups, or Random for charniid. C: Thomas cluster on homogeneous background. $\mathrm{CH}$ : Thomas cluster on heterogeneous background. For the heterogeneous backgrounds, the moving window radius is $0.5 \mathrm{~m}$ since that radius produced the best-fit for Charniids: Heterogeneous Cluster model on a background density constructed from all species: $\left(\mathrm{CH}_{\mathrm{all}}\right)$; Ivesheadiamorphs $\left(\mathrm{CH}_{\text {Ive }}\right)$, Fractofusus $\left(\mathrm{CH}_{\text {Frac }}\right)$, Charniodiscus $\left(\mathrm{CH}_{\mathrm{Cha}}\right)$, Primocandelabrum $\left(\mathrm{CH}_{\text {Primo }}\right)$. CSR on heterogeneous background $(\mathrm{H})$. Bradgatia $\left(\mathrm{H}_{\mathrm{Bra}}\right)$, Lobate Discs $\left(\mathrm{H}_{\mathrm{Lob}}\right)$, Thectardis $\left(\mathrm{H}_{\mathrm{The}}\right)$, Charniid $\left(\mathrm{H}_{\mathrm{Char}}\right) \cdot p_{d}=1$ corresponds to a perfect fit of the model on the data, while $p_{d}=0$ corresponds to no fit. The H14 surface did not possess enough non-Fractofusus specimens to perform similar analyses. NA: not applicable.

\section{Extended Data Table 4: The best-fit double Thomas cluster models fit onto other taxon} $\mathrm{p}_{\mathrm{d}}=1$ corresponds to a perfect fit of the model on the data, while $\mathrm{p}_{\mathrm{d}}=0$ corresponds to no fit. Note, that while these numbers may seem low (such as the 'E' surface fit), they need to be 
426 considered in context of the PCF graph (Extended Data Fig. 2b), which clearly shows a good

427 fit to the data, with the small fluctuations of the observed PCF around the model PCF.

428

429 Extended Data Table 5: Models for bivariate analysis between different size classes of

430 Fractofusus on the H14 surface. SP refers to shared parents models, and LCM refers to

431 linked cluster models. $p_{d}=1$ corresponds to a perfect fit of the model on the data, while $\mathrm{p}_{\mathrm{d}}=$

4320 corresponds to no fit. The large size class was randomly distributed, but was approximated

433 by a cluster model, which was required for input into Programita. ${ }^{9}$

434 